\title{
THE TWO-CENTRE COULOMB PROBLEM IN QUANTUM MECHANICS. AN INFLUENCE OF THE DIMENSIONS
}

\author{
D. I. Bondar ${ }^{1}$, V. Yu. Lazur ${ }^{1}$, I. M. Shvab ${ }^{1}$, S. Chalupka ${ }^{2}$ \\ ${ }^{1}$ Department of Theoretical Physics, Uzhgorod National University, \\ Voloshin Str. 54, 88000 Uzhgorod, Ukraine \\ e-mail:dbondar@univ.uzhgorod.ua \\ e-mail:lazur@univ.uzhgorod.ua \\ shvabi@univ.uzhgorod.ua \\ 2 Institute of Physics, P.J. Šafárik University, Jesenná 5, \\ 04154 Košice, Slovak Republic
}

(Received October 14, 2005; received in final form March 21, 2006)

\begin{abstract}
This work investigates the two-centre Coulomb problem. The analysis is carried out in the context of the dimensional influence $(D \geq 3)$. Asymptotic solutions of the Schrödinger equation with two Coulomb potentials are obtained in the hyperspheroidal coordinate system. These solutions are expressed in the form of confluent Heun functions, particular cases being the Coulomb spheroidal functions. The asymptotic expression for energy for a large intercentre separation of the considered system are obtained and basic properties of the mentioned expressions are studied.

Key words: two-centre Coulomb problem, comparison equation method, influence of the dimensions, Heun's equation.
\end{abstract}

PACS number(s): 02.60.Lj, 02.30.Mv, 02.30.Gp, 03.65.Ge

\section{INTRODUCTION}

This work provides a framework to the investigation of asymptotic eigensolutions of two-centre Coulomb problem in the hyperspheroidal coordinate system. The analysis is based on the ideas which picture the physical system of interest to be embedded in the space of an inflated number of spatial dimensions as an interim step. A strong incentive for this method was provided by its historical precursors. It was shown by Ehrenfest $[1,2]$ that the generalization of physical theories in case of space with the arbitrary dimension $D$ often resulted in a new and unexpected look on the essence of the examined problem. In recent years such an approach has been given a considerable impetus it is also widely used in theoretical physic. $1 / D$-expansion is based on it as well as a novel size scaling - calculation method of mechanics and field theory, which was used, in particular, to research into the properties of atoms in strong electric and magnetic fields [3-6] and into the three-body problem together with many other problems. The discussion on the state of the mentioned method, its different variants and application in the theory of atoms, molecules and quantum chemistry may be found in [6].

This work is devoted to a generalization of the results of the asymptotic theory for the quantum mechanics two-centre Coulomb problem $Z_{1} e Z_{2}[7,8]$ by inflating the number of spatial dimension. We assume asymptotic expansions with the help of the comparison equation method of the Schrödinger equation for the $D$ dimensional two-centre Coulomb problem (briefly the $\left(Z_{1} e Z_{2}\right)_{D}$ problem) for a large intercentre separation

$$
\left[-\frac{1}{2} \Delta-\frac{Z_{1}}{r_{1}}-\frac{Z_{2}}{r_{2}}\right] \Psi(\mathbf{r} ; R)=E \Psi(\mathbf{r} ; R)
$$

in the hyperspheroidal coordinate system which corre- sponds at $(D \geq 3)$ to the confocal prolate rotational ellipsoids and two-sheets rotational hyperboloids. Here $r_{1}, r_{2}$ are distances from an electron to the charges $Z_{1}$ and $Z_{2}$, respectively, and $R$ is the separation of centres, $\hbar=m_{e}=e=1$. After separating (1.1) in the hyperspheroidal coordinates, we may state that the $\left(Z_{1} e Z_{2}\right)_{D}$ problem leads to two-coupled confluent Heun equations $[8,9]$.

The solution of the Schrödinger equation with twocentre potential is of considerable interest in various problems with few-body systems [7]. They describe the bound states of light particles in the field of two heavy particles. Usually such a type of system arises in molecular physics. However, in recent years there has been an interest of other systems modelling by the two-centre Schrödinger equation, namely baryons containing heavy quarks (QQg baryons) [10] and heavy flavoured hybrid mesons (QQg mesons) are now becoming the subject of extensive investigation. There is a close connection between the two-centre Coulomb problem $\left(Z_{1} e Z_{2}\right)_{D}$ and $S U(2)$ monopole [11,12]. A five-dimensional bound system of "charge-dion" with $S U(2)$ - Yang monopole [13] is described by equations which we obtain at the separation of variables of (1.1) in hyperspheroidal coordinates. Besides, equation (1.1) has a reference to a well known Teukolsky equation [14].

The outline of the paper is as follows. In section II hyperspheroidal coordinates are considered in terms of which the wave function is separated. This serves as a vehicle for deriving equations for the radial and angular Coulomb hyperspheroidal functions. Section III treats asymptotic expansion of Coulomb hyperspheroidal functions for a large parameter $p$. Section IV turns to a solution of equations (2.16) and (2.17). In section $\mathrm{V}$ we obtain the expansions of energy for large centre separations $R$ and a quasi-crossing of energetic terms is studied. 
In section VI the rules of correspondences of electronic terms in the limits of $R \rightarrow 0$ and $R \rightarrow \infty$ are considered.

\section{SEPARATION OF VARIABLES IN SCHRÖDINGER EQUATION OF THE TWO-CENTRE COULOMB PROBLEM IN SPACES OF ARBITRARY DIMENSION}

We introduce in the $D$-dimensional Euclidean space $E_{D}$ the hyperspheroidal coordinate system with the origin in the midpoint of the segment $R$ and foci in its endpoints:

$$
\begin{aligned}
& x_{1}=\frac{R}{2} \cosh u \cos v, \\
& x_{2}=\frac{R}{2} \sinh u \sin v \cos \beta_{D-2}, \\
& x_{3}=\frac{R}{2} \sinh u \sin v \sin \beta_{D-2} \cos \beta_{D-3}, \\
& \vdots \\
& x_{D-1}=\frac{R}{2} \sinh u \sin v \sin \beta_{D-2} \ldots \sin \beta_{2} \cos \beta_{1}, \\
& x_{D}=\frac{R}{2} \sinh u \sin v \sin \beta_{D-2} \ldots \sin \beta_{2} \sin \beta_{1} .
\end{aligned}
$$

Here change in the following limits:

$$
\begin{aligned}
& 0 \leq v<2 \pi, \quad \text { if } \quad D=2, \\
& 0 \leq v<\pi, \quad \text { if } \quad D>2, \\
& 0 \leq u<\infty, \quad 0 \leq \beta_{1}<2 \pi \\
& \beta_{0}=0, \quad k=2,3, \ldots, D-2 . \\
& 0 \leq \beta_{k}<\pi, \quad k
\end{aligned}
$$

The hyperspheroidal coordinate system (2.2) is the special case of the polyspheroidal coordinate system [15]. The prolate spheroidal coordinate system [7] is (at $D=$ 3) a special case of the system (2.2).

Surfaces on which $u$ and $v$ are constant for the case $D \geq 3$ represent confocal rotational ellipsoids and hyperboloids, respectively,

$$
\begin{aligned}
& \frac{x_{1}^{2}}{(R / 2)^{2} \cosh ^{2} u}+\frac{\left(x_{2}^{2}+\ldots+x_{D}^{2}\right)}{(R / 2)^{2} \sinh ^{2} u}=1, \\
& \frac{x_{1}^{2}}{(R / 2)^{2} \cos ^{2} v}-\frac{\left(x_{2}^{2}+\ldots+x_{D}^{2}\right)}{(R / 2)^{2} \sin ^{2} v}=1 .
\end{aligned}
$$

In the case of $D=2$ these surfaces degenerate in confocal ellipses or hyperboloids, respectively. The arc element formula in the coordinate system $(2.2)$ is

$$
d s^{2}=h_{u}^{2} d u^{2}+h_{v}^{2} d v^{2}+\sum_{k=1}^{D-2} h_{\beta_{k}}^{2} d \beta_{k}=\frac{R^{2}}{4}\left(\cosh ^{2} u-\cos ^{2} v\right)\left(d u^{2}+d v^{2}\right)+\frac{R^{2}}{4} \sinh ^{2} u \sin ^{2} v d s_{D-2}^{2}
$$

where scale coefficients

$$
\begin{aligned}
& h_{u}=h_{v}=\frac{R}{2} \sqrt{\cosh ^{2} u-\cos ^{2} v}, \\
& h_{\beta_{k}}=\frac{R}{2} \sinh u \sin v \prod_{i=k+1}^{D-2} \sin \beta_{i}, \\
& h_{\beta_{D-2}}=\frac{R}{2} \sinh u \sin v,
\end{aligned}
$$

$k=1,2, \ldots, D-3$ and $d s_{D-2}$ is the line element on the $(D-2)$ dimensional unit sphere $($ at $D>3)$ or the unit circle (for $D=3$ )

$$
\begin{aligned}
d s_{D-2}^{2} & =d \beta_{D-2}^{1}+\sin ^{2} \beta_{D-2} d \beta_{D-3}^{2}+, \ldots, \\
& +\sin ^{2} \beta_{D-2} \ldots \sin ^{2} \beta_{2} d \beta_{1}^{2} .
\end{aligned}
$$

Further, it is suitable to link hyperspheroidal coordinates (2) with the distances $r_{1}$ and $r_{2}$ from points $\mathbf{r}=\left(x_{1}, x_{2}, \ldots, x_{D}\right)$ to the left and right focus, respectively. Both the foci are placed on the $x_{1}$ axis. Then

$$
\begin{aligned}
& r_{1}=\sqrt{\left(x_{1}+\frac{R}{2}\right)^{2}+\sum_{i=2}^{D} x_{i}^{2}}=\frac{R}{2}(\cosh u+\cos v), \\
& r_{2}=\sqrt{\left(x_{1}-\frac{R}{2}\right)^{2}+\sum_{i=2}^{D} x_{i}^{2}}=\frac{R}{2}(\cosh u-\cos v) .
\end{aligned}
$$

According to (2.6) equation (1.1) has the form

$$
\begin{aligned}
& \left\{\frac{1}{\cosh ^{2} u-\cos ^{2} v}\left[\frac{1}{\sinh ^{D-2} u} \frac{\partial}{\partial u} \sinh ^{D-2} u \frac{\partial}{\partial u}+\frac{1}{\sin ^{D-2} v} \frac{\partial}{\partial v} \sin ^{D-2} v \frac{\partial}{\partial v}\right]\right. \\
& \left.+\frac{1}{\sinh ^{2} u \sin ^{2} v H_{\beta}} \sum_{k=1}^{D-2} \frac{\partial}{\partial \beta_{k}}\left(\frac{H_{\beta}}{H_{\beta_{k}}^{2}}\right) \frac{\partial}{\partial \beta_{k}}+R\left(\frac{Z_{1}}{\cosh u+\cos v}+\frac{Z_{2}}{\cosh u-\cos v}\right)+\frac{R^{2} E}{2}\right\} \Psi=0,
\end{aligned}
$$


where

$$
\begin{aligned}
H_{\beta}= & \prod_{k=1}^{D-2} H_{\beta_{k}}, \quad H_{\beta_{k}}=\prod_{i=k+1}^{D-2} \sin \beta_{i}, \\
& k=1,2, \ldots, D-3, \quad H_{\beta_{D-2}}=1 .
\end{aligned}
$$

In equation (2.10) the variables are separated by the following substitution

$$
\Psi(\mathbf{r})=\Xi(v) \Pi(u) F\left(\beta_{1}, \beta_{1}, \ldots, \beta_{D-2}\right),
$$

where

$$
F\left(\beta_{1}, \beta_{1}, \ldots, \beta_{D-2}\right)=\prod_{k=1}^{D-2} F_{k}\left(\beta_{k}\right),
$$

and we come to the necessity to solve the following system of equations in region (2.3):

$$
\begin{aligned}
& {\left[\frac{1}{\sinh ^{D-2} u} \frac{\partial}{\partial u} \sinh ^{D-2} u \frac{\partial}{\partial u}-\frac{m_{D-2}\left(m_{D-2}+D-3\right)}{\sinh ^{2} u}+a \cosh u+p^{2}\left(\cosh ^{2} u-1\right)-\lambda\right] \Pi=0,} \\
& {\left[\frac{1}{\sin ^{D-2} v} \frac{\partial}{\partial v} \sin ^{D-2} v \frac{\partial}{\partial v}-\frac{m_{D-2}\left(m_{D-2}+D-3\right)}{\sin ^{2} v}+b \cos v-p^{2}\left(1-\cos ^{2} v\right)+\lambda\right] \Xi=0,} \\
& {\left[\frac{\partial^{2}}{\partial \beta_{1}^{2}}+m_{1}^{2}\right] F_{1}\left(\beta_{1}\right)=0,} \\
& {\left[\frac{1}{\sin ^{k-1} \beta_{k}} \frac{\partial}{\partial \beta_{k}} \sin ^{k-1} \beta_{k} \frac{\partial}{\partial \beta_{k}}-\frac{m_{k-1}\left(m_{k-1}+k-2\right)}{\sin ^{2} \beta_{k}}+m_{k}\left(m_{k}+k-1\right)\right] F_{k}\left(\beta_{k}\right)=0, \quad k=2,3, \ldots, D-2 .}
\end{aligned}
$$

Here $\lambda, m_{1}, m_{2}, \ldots, m_{D-2}$ are the separations constants and

$p=\frac{R}{2} \sqrt{-2 E}, a=R\left(Z_{1}+Z_{2}\right), b=R\left(Z_{2}-Z_{1}\right)$.

In particular, for $D=2$ is $m_{0}=0$ and equations (2.16), (2.17) are missing.

In restricting further deductions to the bound states $E<0$ (a discrete spectrum) it is seen that $0 \leq p<\infty$. Solutions of the equations will be referred to as solutions of the $p$-type. Otherwise said, $E>0$ (a continuous spectrum), $p=i c$ and $0 \leq c<\infty$. These solutions are of the $c$-type.

It is expedient now to introduce new variables $\xi, \eta$ by the formulae

$$
\begin{array}{ll}
\xi=\cosh u, & 1 \leq \xi<\infty, \\
\eta=\cos v, & -1 \leq \eta \leq 1,
\end{array}
$$

which are valid only in the case $D \geq 3$. Equations (2.15) and (2.14) then yield

$$
\begin{aligned}
& \frac{1}{\left(\xi^{2}-1\right)^{\frac{D-3}{2}}} \frac{d}{d \xi}\left(\xi^{2}-1\right)^{\frac{D-1}{2}} \frac{d \Pi}{d \xi}+\left[-\lambda-p^{2}\left(\xi^{2}-1\right)+a \xi-\frac{m_{D-2}\left(m_{D-2}+D-3\right)}{\xi^{2}-1}\right] \Pi=0 \\
& \frac{1}{\left(1-\eta^{2}\right)^{\frac{D-3}{2}}} \frac{d}{d \eta}\left(1-\eta^{2}\right)^{\frac{D-1}{2}} \frac{d \Xi}{d \eta}+\left[\lambda-p^{2}\left(1-\eta^{2}\right)+b \eta-\frac{m_{D-2}\left(m_{D-2}+D-3\right)}{1-\eta^{2}}\right] \Xi=0 .
\end{aligned}
$$

Both equations (2.19) and (2.20) are confluent Heun equations $[8,9]$.

In the case of a discrete spectrum, $\Xi(\eta)$ and $\Pi(\xi)$ obey the boundary conditions

$$
\begin{gathered}
|\Pi(1)|<\infty, \quad|\Pi(\xi)| \stackrel{\xi \rightarrow \infty}{\longrightarrow} 0 . \\
|\Xi( \pm 1)|<\infty,
\end{gathered}
$$

we introduce radial Coulomb hyperspheroidal functions of $p$-type $\Pi_{m_{D-2} k}^{(D)}(p, a ; \xi)$ as solutions of Sturm-Liouville problem $(2.19),(2.21)$ on the ray $\xi \in[1, \infty)$, where $k$ is the number of zeros inside $[1, \infty)$.

Also let us introduce angular Coulomb hyperspheroidal functions of $p$-type $\Xi_{m_{D-2} q}^{(D)}(p, b ; \eta)$ as solutions of Sturm-Liouville problem $(2.20),(2.22)$ on the interval $\eta \in[-1,1]$, where $q$ is the number of zeros inside $[-1,1]$.

The transformations 


$$
\begin{aligned}
& \Pi_{m_{D-2} q}^{(D)}(p, a ; \xi)=\frac{U(\xi)}{\left(\xi^{2}-1\right)^{\frac{D-1}{4}}}, \\
& \Xi_{m_{D-2} q}^{(D)}(p, b ; \eta)=\frac{V(\eta)}{\left(1-\eta^{2}\right)^{\frac{D-1}{4}}},
\end{aligned}
$$

change equations (2.19), (2.20) into

$$
\begin{aligned}
& {\left[\frac{d^{2}}{d \xi^{2}}-p^{2}+\frac{a \xi-\lambda^{\prime}}{1-\xi^{2}}+\frac{1-\mu^{2}}{\left(\xi^{2}-1\right)^{2}}\right] U(\xi)=0} \\
& {\left[\frac{d^{2}}{d \eta^{2}}-p^{2}+\frac{b \eta+\lambda^{\prime}}{1-\eta^{2}}+\frac{1-\mu^{2}}{\left(1-\eta^{2}\right)^{2}}\right] V(\eta)=0 .}
\end{aligned}
$$

Here

$$
\lambda^{\prime}=\lambda+\frac{(D-1)(D-3)}{4}, \mu=m_{D-2}+\frac{D-3}{2} .
$$

Equations (2.25), (2.26) can be used as the starting point of a different asymptotic expansion.

\section{ASYMPTOTIC EXPANSIONS FOR} COULOMB HYPERSPHEROIDAL FUNCTIONS AND THE SEPARATION CONSTANT

The procedure form finding an asymptotic solution of the $\left(Z_{1} e Z_{2}\right)_{D}$ problem for a large parameter $R$ re- quires our knowledge of the asymptotic expression for $\Pi_{m_{D-2} k}^{(D)}(p, 2 p \alpha ; \xi)$ and $\Xi_{m_{D-2} q}^{(D)}(p, 2 p \beta ; \eta)$

$$
\begin{aligned}
& \Pi_{m_{D-2} k}^{(D)}(p, 2 p \alpha ; \xi), \quad p \rightarrow \infty, \quad \mu=O(1), \\
& k=O(1), \quad \alpha=O(1), \\
& \Xi_{m_{D-2} q}^{(D)}(p, 2 p \beta ; \eta), \quad p \rightarrow \infty, \quad \mu=O(1), \\
& q=O(1), \quad \beta=O(1),
\end{aligned}
$$

where

$$
\begin{aligned}
\nu & =\frac{\lambda^{\prime}}{2 p}, \quad \beta=\frac{b}{2 p}=\frac{Z_{2}-Z_{1}}{\sqrt{-2 E}}, \\
\alpha & =\frac{a}{2 p}=\frac{Z_{2}+Z_{1}}{\sqrt{-2 E}} .
\end{aligned}
$$

If conditions (3.28) and (3.29) are satisfied then an estimate $\nu=O(1)$.

Now we rewrite equations $(2.25),(2.26)$ suing relation (3.30). Then

$$
\begin{aligned}
& \frac{d^{2} U}{d \xi^{2}}+\left[-p^{2}+\frac{2 p(\alpha \xi-\nu)}{\xi^{2}-1}+\frac{1-\mu^{2}}{\left(\xi^{2}-1\right)^{2}}\right] U(\xi)=0 \\
& \frac{d^{2} V}{d \eta^{2}}+\left[-p^{2}+\frac{2 p(\beta \eta+\nu)}{1-\eta^{2}}+\frac{1-\mu^{2}}{\left(1-\eta^{2}\right)^{2}}\right] V(\eta)=0 .
\end{aligned}
$$

In virtue of the normalization of $\Psi(\mathbf{r}, R)$ as can be seen from (2.24) and (2.23), it is necessary to satisfy the boundary conditions

$$
\begin{gathered}
\left.U(\xi)\right|_{\xi=1}=0,\left.\quad U(\xi)\right|_{\xi \rightarrow \infty}=0, \\
\left.V(\eta)\right|_{\eta= \pm 1}=0 .
\end{gathered}
$$

The normalization of $U(\xi), V(\eta)$ result from the normalization of the relating Coulomb hyperspheroidal functions. Then

$$
\begin{aligned}
& \int_{1}^{\infty}\left(\xi^{2}-1\right)^{\frac{1-D}{2}} U^{2}(\xi) d \xi=1 \\
& \int_{-1}^{1}\left(1-\eta^{2}\right)^{\frac{1-D}{2}} V^{2}(\eta) d \eta=1 .
\end{aligned}
$$

We further consider equation (3.32). The asymptotic solution is constructed in two overlapping intervals

$$
\Omega_{-}=\left[-1, \eta_{1}\right], \quad \Omega_{+}=\left[\eta_{2}, 1\right], \quad \eta_{-}<\eta_{2}<\eta_{1}<\eta_{+},
$$

where $\eta_{-}=-1+(-\beta+\nu) / p, \quad \eta_{+}=1-(\beta+\nu) / p$ are turning points.
In the region of $\Omega_{-}$the solution of the equation is expressed in a sophisticated form (comparison equation method)

$$
V_{-}(\eta)=N_{-}\left[u_{-}^{\prime}(\eta)\right]^{-1 / 2} M_{\chi, \frac{\mu}{2}}\left[2 p u_{-}(\eta)\right]
$$

where $N_{-}$is the normalization constant and function $M_{\chi, \frac{\mu}{2}}(z)$ satisfying Whittaker's differential equation is regular at $z=0$ ( [16], [17] vol. 1) The index $\chi$ has not been determined yet. To satisfy conditions (3.34) at $\eta=-1$ it should be sufficient that $\left.u_{-}(\eta)\right|_{\eta=-1}=0$.

We assume that function $u_{-}(\eta)$ and the spectral parameter $\lambda_{\mu q}^{\prime(n)}$ are expressed as descending series in $p$, thus

$$
\begin{gathered}
u_{-}(\eta)=\sum_{i=0}^{\infty} \frac{u_{i}}{p^{i}}, \\
\lambda_{\mu q}^{\prime(\eta)}(p)=2 p \nu(p)=2 p \sum_{i=0}^{\infty} \frac{\nu_{i}}{p^{i}} .
\end{gathered}
$$

Applying the recurrent procedure [7], we find the scaling transformation 


$$
\begin{aligned}
& u_{-}(p, \beta, \chi ; \eta)=1+\eta+\frac{1}{p}\left\{(\chi+\beta) \ln \left(\frac{1-\eta}{2}\right)\right\} \\
& +\frac{1}{p^{2}}\left\{\frac{\chi(\chi+\beta)}{1+\eta} \ln \left(\frac{1-\eta}{2}\right)-\frac{(\chi+\beta)^{2}+\tau}{2(1-\eta)}+\frac{1}{4}\left[(\chi+\beta)^{2}+\tau\right]+\frac{1}{2} \chi(\chi+\beta)\right\} \\
& +\frac{1}{p^{3}}\left\{-\frac{\chi(\chi+\beta)^{2}}{\left(1+\eta^{2}\right)} \ln ^{2}\left(\frac{1-\eta}{2}\right)+\frac{\left(3 \chi^{2}+\tau\right)(\chi+\beta)}{2(1+\eta)^{2}} \ln \left(\frac{1-\eta}{2}\right)-\frac{\chi\left[(\chi+\beta)^{2}+\tau\right]}{2(1-\eta)}\right. \\
& -\frac{(\chi+\beta)\left[(\chi+\beta)^{2}+\tau+1\right]}{4(1-\eta)^{2}}+\frac{\left(3 \chi^{2}+\tau\right)(\chi+\beta)}{4(1+\eta)} \\
& \left.+\frac{1}{16}\left(10 \chi^{3}+18 \chi^{2} \beta+9 \chi \beta^{2}+2 \tau \beta+\beta^{3}+6 \chi \tau+\chi+\beta\right)\right\}+O\left(p^{-4}\right),
\end{aligned}
$$

where $\tau=\left(1-\mu^{2}\right) / 4$.

From the conditions of the solvability of the recurrent process we obtain the asymptotic expansion for the eigenvalues $\lambda_{\mu q}^{\prime(\eta)}$ which correspond to the left centre

$$
\begin{aligned}
& \lambda_{\mu q}^{\prime(\eta)}(p, 2 p \beta ;-1, \chi)=2 p(2 \chi+\beta)-\left\{2 \chi \beta+\frac{1}{2}\left(4 \chi^{2}+1-\mu^{2}\right)\right\} \\
& -\frac{1}{8 p}\left\{2 \chi\left(4 \chi^{2}-\mu^{2}+1\right)+\left(12 \chi^{2}+1-\mu^{2}\right) \beta+4 \chi \beta^{2}\right\}+\frac{1}{64 p^{2}}\left\{-80 \chi^{4}-40 \chi^{2}+24 \chi^{2} \mu^{2}\right. \\
& \left.-\left(1-\mu^{2}\right)^{2}-\left(80 \chi^{2}+20-12 \mu^{2}\right) 2 \chi \beta-\left(96 \chi^{2}+8\left(1-\mu^{2}\right)\right) \beta^{2}-16 \chi \beta^{3}\right\}+O\left(\frac{1}{p^{3}}\right)
\end{aligned}
$$

Near the right centre, the solution $V_{+}(\eta)$ of the angular equation (3.32) is analogical from (3.37) but with another parameter $\chi^{\prime}$ and with a different scale transformation $u_{+}(\eta)$. Transition form the left centre to the right one in expansions (3.40) and (3.41) is carried out by replacements

$$
\begin{gathered}
\eta \rightarrow-\eta, \quad \beta \rightarrow-\beta, \quad \chi \rightarrow \chi^{\prime}, \\
u_{+}\left(p, \beta, \chi^{\prime} ; \eta\right)=u_{-}(p,-\beta, \chi ;-\eta), \\
\lambda_{\mu q}^{(\eta)}\left(p, 2 p \beta ; 1, \chi^{\prime}\right)=\lambda_{\mu q}^{\prime(n)}(p,-2 p \beta ;-1, \chi) .
\end{gathered}
$$

Thus, constructed asymptotic expansions for the eigenvalues $\lambda^{\prime(\eta)}\left(p, 2 p \beta ; 1, \chi^{\prime}\right)$ and $\lambda_{\mu q}^{\prime(n)}(p,-2 p \beta ;-1, \chi)$ depend on $\chi$ and $\chi^{\prime}$. Equating these expansions we obtain

$$
\chi^{\prime}=\chi+\beta
$$

On the overlapping interval $\Omega_{-} \bigcap \Omega_{+}\left(\eta_{2}<\eta<\eta_{1}\right)$ we demand

$$
V_{-}(\eta)=C V_{+}(\eta), \quad(C=\text { const })
$$

It means is zero of the Wronskian of $V_{-}$and $V_{+}$

$$
V_{-}^{\prime}(\eta) V_{+}(\eta)-V_{-}(\eta) V_{+}^{\prime}(\eta)=0
$$

Substituting the found expansions of the function $V_{-}(\eta)$ and $V_{+}(\eta)$ in (3.45) and using an asymptotic expansions of the Whittaker's function $[7,16]$ for a large argument leads to the transcendent equation which connects $\chi$ and $\chi^{\prime}$

$$
\begin{aligned}
& {\left[\frac{1}{\pi} \tan \left(\pi\left(\chi-\frac{\mu+1}{2}\right)\right)\right]\left[\frac{1}{\pi} \tan \left(\pi\left(\chi^{\prime}-\frac{\mu+1}{2}\right)\right)\right]} \\
& =\frac{(4 p)^{2\left(\chi+\chi^{\prime}\right)} e^{-4 p}}{\Gamma\left(\chi+\frac{1+\mu}{2}\right) \Gamma\left(\chi+\frac{1-\mu}{2}\right) \Gamma\left(\chi^{\prime}+\frac{1+\mu}{2}\right) \Gamma\left(\chi^{\prime}+\frac{1-\mu}{2}\right)}\left[1+O\left(\frac{1}{p}\right)\right] .
\end{aligned}
$$

We replace the right of (3.46) by $\delta^{2}$. Since $\delta^{2}$ exponentially tends to zero as $p \rightarrow \infty$ systems (3.43), (3.46) can be solved by successive approximation. If we neglect small term $\delta^{2}$, then we obtain two series solutions which correspond to the vanishing of one of the tangents in
(3.46). Then they are either

$$
\chi_{0}=n_{2}+(1+\mu) / 2, \quad \chi_{0}^{\prime}=n_{2}+(1+\mu) / 2+\beta,
$$


or

$$
\begin{gathered}
\chi_{0}^{\prime}=n_{2}^{\prime}+(1+\mu) / 2, \quad \chi_{0}^{\prime}=n_{2}^{\prime}+(1+\mu) / 2-\beta, \\
\chi=\chi_{0}+\delta \chi, \quad \chi^{\prime}=\chi_{0}^{\prime}+\delta \chi^{\prime} .
\end{gathered}
$$

Here $n_{2}, n_{2}^{\prime}$ are non-negative integers.
The method of the calculation of the first iterations of (3.43), (3.46) depends on whether $\beta$ is near the integer or not. If the values of $\beta$ are not in the vicinity of the integers, then corrections $\delta \chi$ and $\delta \chi^{\prime}$ can be found independently. For $\beta$ near small neighborhoods of integers $\left(\beta \approx n_{2}^{\prime}-n_{2}\right)$ we may calculate $\delta \chi$ from (3.46):

$$
\begin{gathered}
\delta \chi=-\frac{1}{2 \pi} \tan \pi \beta \pm \sqrt{\left(\frac{1}{2 \pi} \tan \pi \beta\right)^{2}+\delta^{2}\left(n_{2}, n_{2}^{\prime}, \mu, p\right)}, \\
\delta \chi^{\prime}=\frac{1}{2 \pi} \tan \pi \beta \pm \sqrt{\left(\frac{1}{2 \pi} \tan \pi \beta\right)^{2}+\delta^{2}\left(n_{2}, n_{2}^{\prime}, \mu, p\right)},
\end{gathered}
$$

where

$$
\delta\left(n_{2}, n_{2}^{\prime}, \mu, p\right)=\frac{4 p^{n_{2}+n_{2}^{\prime}+\mu+1} e^{-2 p}}{\sqrt{n_{2} ! \Gamma\left(n_{2}+\mu+1\right) n_{2}^{\prime} ! \Gamma\left(n_{2}^{\prime}+\mu+1\right)}}\left\{1-\frac{1}{4 p}\left[\chi_{0}^{2}+\chi_{0}^{\prime 2}+4 \chi_{0} \chi_{0}^{\prime}+\frac{1-\mu^{2}}{2}\right]+O\left(\frac{1}{p^{2}}\right)\right\} .
$$

The asymptotic expansion of the first order with respect to $p$ of $\Xi_{m_{D-2} q}^{(D)}(p, 2 p \beta ; \eta)$, satisfying conditions (3.36) and (3.44) in the region $\Omega_{-}$, has the form:

$$
\Xi_{m_{D-2} q}^{(D)}(p, 2 p \beta ; \eta)=d_{-} N_{-} \frac{M_{\chi, \frac{m_{D-2}}{2}+\frac{D-3}{4}}\left[2 p(1+\eta)+2(\chi+\beta) \ln \frac{1-\eta}{2}\right]}{\left(1-\eta^{2}\right)^{\frac{D-1}{4}}}[1+O(1 / p)], \quad \eta \in \Omega_{-},
$$

where

$$
\begin{aligned}
\frac{1}{N_{-}^{2}} & =\frac{p^{\frac{D-3}{2}}}{2}\left[\frac{\Gamma\left(\frac{3-D}{2}+1\right) \Gamma\left(m_{D-2}+\frac{D-3}{2}+1\right) \Gamma\left(\chi+\frac{1-m_{D-2}}{2}-\frac{D-3}{4}\right)}{\Gamma\left(\chi+\frac{1+m_{D-2}}{2}+\frac{D-3}{4}\right)}\right]^{2} \\
& \times \sum_{j=0}^{n} \frac{\Gamma\left(m_{D-2}+j+1\right)}{\Gamma(j+1) \Gamma^{2}\left(\chi+\frac{1-m_{D-2}}{2}-\frac{D-3}{4}-j\right)} \frac{1}{\Gamma^{2}\left(\frac{3-D}{2}-n+j+1\right)},
\end{aligned}
$$

and $n=\chi-\frac{1+m_{D-2}}{2}+\frac{3-D}{4}$.

In similar manner (by changing $\chi \rightarrow \chi^{\prime}, N_{-} \rightarrow N_{+}$) for $\eta \in \Omega_{+}$we can deduce that

$$
\Xi_{m_{D-2} q}^{(D)}(p, 2 p \beta ; \eta)=d_{+} N_{+} \frac{M_{\chi, \frac{m_{D-2}}{2}+\frac{D-3}{4}}\left[\left(2 p(1-\eta)+2\left(\chi^{\prime}-\beta\right) \ln \frac{1+\eta}{2}\right]\right.}{\left(1-\eta^{2}\right)^{\frac{D-1}{4}}}[1+O(1 / p)], \quad \eta \in \Omega_{+},
$$

where we $N_{+}$obtain from $N_{-}$by the replacement $\chi \rightarrow \chi^{\prime}$. The coefficients $d_{+}$and $d_{-}$are determined by relations

$$
d_{+}^{2}+d_{-}^{2}=1
$$

$$
\begin{aligned}
d_{-}= & \sqrt{\left|\frac{\sin \left[\pi\left(2 \chi^{\prime}-1-m_{D-2}+\frac{3-D}{2}\right)\right]}{\sin \left[\pi\left(2 \chi-1-m_{D-2}+\frac{3-D}{2}\right)\right]+\sin \left[\pi\left(2 \chi^{\prime}-1-m_{D-2}+\frac{3-D}{2}\right)\right]}\right|} \\
& \times \operatorname{sgn}\left(\frac{-\cos \left[\pi\left(\chi-\frac{1+m_{D-2}}{2}+\frac{3-D}{4}\right)\right]}{\sin \left[\pi\left(\chi^{\prime}-\frac{1+m_{D-2}}{2}+\frac{3-D}{4}\right)\right]}\right), \\
d_{+}= & \sqrt{\left|\frac{\sin \left[\pi\left(2 \chi-1-m_{D-2}+\frac{3-D}{2}\right)\right]}{\sin \left[\pi\left(2 \chi-1-m_{D-2}+\frac{3-D}{2}\right)\right]+\sin \left[\pi\left(2 \chi^{\prime}-1-m_{D-2}+\frac{3-D}{2}\right)\right]}\right|},
\end{aligned}
$$


where $\operatorname{sgn}(x)$ is the sign function.

A solution $U(\xi)$ of (3.31) will be searched in the form analogous to (3.37):

$$
U(\xi)=N\left[\omega^{\prime}(\xi)\right]^{-1 / 2} M_{\sigma, \mu / 2}[2 p \omega(\xi)] .
$$

It follows from condition (3.33) of decreasing as $\xi \rightarrow \infty$

$$
\sigma=k+(1+\mu) / 2, \quad k=0,1,2, \ldots,
$$

where $k$ is the number of zeros of the radial hyperspheroidal functions. The Whittaker's function satisfying (3.60) may be expressed by Laguerre polynomial [16].

If we change

$$
\xi \rightarrow-\eta, \quad p \rightarrow-p, \quad \alpha \rightarrow-\beta,
$$

then in the neighborhood $\xi=1$ with conditions $U(1)=0$ equation (3.31) transforms into equation (3.32) with $V(-1)=0$ in the vicinity $\eta=-1$. Further, from (3.40) and (3.41) follows expansion for the scale transformations $\omega(\xi)$ and the eigenvalue $\lambda^{\prime(\xi)}$, respectively

$\lambda_{\mu k}^{\prime(\xi)}(p, 2 p \alpha)=\lambda_{\mu k}^{\prime(\eta)}\left(-p,-2 p \alpha ;-1, k+\frac{\mu+1}{2}\right)$.

The desired asymptotic expansion of $\Pi_{m_{D-2} k}^{(D)}(p, 2 p \alpha ; \xi)$ for which condition (3.35) holds, id:

$$
\Pi_{m_{D-2} k}^{(D)}(p, 2 p \alpha ; \xi)=N \frac{M_{\sigma, \frac{m_{D-2}}{2}+\frac{D-3}{4}}[2 p(\xi-1)]}{\left(\xi^{2}-1\right)^{\frac{D-1}{4}}}\left[1+O\left(\frac{1}{p}\right)\right],+1 \leq \xi<\infty
$$

whereupon

$$
\frac{1}{N^{2}}=\frac{p^{\frac{D-3}{2}}}{2}\left[\frac{\Gamma\left(\frac{3-D}{2}+1\right) \Gamma\left(m_{D-2}+\frac{D-3}{2}+1\right) k !}{\Gamma\left(k+m_{D-2}+\frac{D-3}{2}+1\right)}\right]^{2} \sum_{j=0}^{k} \frac{\left(m_{D-2}+j\right) !}{j !(k-j) !^{2}} \frac{1}{\Gamma^{2}\left(\frac{3-D}{2}-k+j+1\right)} .
$$

\section{SOLUTION OF EQUATIONS (2.16), (2.17)}

Constants $m_{D-2}\left(m_{D-2}+D-3\right)$ in equations (2.16) and (2.17) represent eigenvalues of the Laplace operator on the $(D-2)$ dimensional unit sphere $S^{D-2}(D>$ $3)$ or the unit circle $(D=3)$. If $m_{1}, m_{2}, \ldots, m_{D-2}$ are non-negative integer and $m_{1} \leq m_{2} \leq, \ldots, m_{D-2}$ then solutions of equations (2.16) and (2.17) are continuous and single-valued, and have the form [15]

$$
\begin{aligned}
& F\left(\beta_{1}\right)=\frac{\exp \left( \pm i m_{1} \beta_{1}\right)}{\sqrt{2 \pi}}, \quad D=3, \\
& F\left(\beta_{1}, \ldots, \beta_{D-2}\right)=\frac{\exp \left( \pm i m_{1} \beta_{1}\right)}{\sqrt{2 \pi}} \\
& \times \prod_{j=2}^{D-2} A_{j} \sin ^{m_{j-1}} \beta_{j} C_{m_{j}-m_{j-1}}^{m_{j-1}+\frac{j-1}{2}}\left(\cos \beta_{j}\right), D>3,
\end{aligned}
$$

where $C_{n}^{m}\left(\cos \beta_{j}\right)$ are Gegenbauer polynomials ( [17] vol. $1,2)$, and $A_{j}$ are normalized constants. From the normalization condition

$$
\int \mid F\left(\beta_{1}, \beta_{2}, \ldots,\left.\beta_{D-2}\right|^{2} d \Omega=1,\right.
$$

where $d \Omega$ is the surface element of the unit sphere, we can find $A_{j}$ :

$$
A_{j}^{2} \int_{-1}^{1}\left(1-t^{2}\right)^{\frac{w-1}{2}}\left[C_{g}^{w / 2}(t)\right]^{2} d t=1
$$

where $t=\cos \beta_{j}$ and

$$
\frac{w}{2}=m_{j-1}+\frac{j-1}{2}, \quad g=m_{j}-m_{j-1} .
$$

After integration we obtain

$$
A_{j}=\sqrt{\frac{g !(w+2 g) \Gamma^{2}(w / 2)}{2^{2-w} \pi \Gamma(g+w)}}=\sqrt{\frac{\left(m_{j}-m_{j-1}\right) !\left(2 m_{j}+j-1\right) \Gamma^{2}\left(m_{j-1}+\frac{j-1}{2}\right)}{2^{3-j-2 m_{j-1}} \pi \Gamma\left(m_{j}+m_{j-1}+j-1\right)}} .
$$




\section{ASYMPTOTIC EXPANSION OF ENERGETIC TERMS. QUASI-CROSSING OF ENERGETIC TERMS}

In such a limiting case as $R \rightarrow \infty$, the hyperspheroidal coordinates near $\xi=1$ and $\eta= \pm 1$ go to the $D$ dimensional parabolic coordinates [18]. Then the problem $\left(Z_{1} e Z_{2}\right)_{D}$ decays into two one-centre Coulomb problems with $Z_{1}$ and $Z_{2}$ charges. Each of the atoms $e Z_{1}$ and $e Z_{2}$ is characterized by a set of quantum numbers $\left[n, n_{1}, n_{2}, \mathbf{m}\right]$ and $\left[n^{\prime}, n_{1}^{\prime}, n_{2}^{\prime}, \mathbf{m}\right]$, which are linked in the following manner:
$n=n_{1}+n_{2}+m_{D-2}+1, \quad n^{\prime}=n_{1}^{\prime}+n_{2}^{\prime}+m_{D-2}+1$,

where $\mathbf{m}=\left[m_{1}, m_{2}, \ldots, m_{D-2}\right]$.

The expansion of the energy in powers $R^{-1}$ is derived by equating $\lambda^{\prime(\xi)}$ and $\lambda^{\prime(\eta)}$

$$
\lambda_{\mu q}^{\prime(\eta)}(p, 2 p \beta)=\lambda_{\mu k}^{\prime(\xi)}(p, 2 p \alpha) .
$$

Taking into account (3.30), (3.41) and (3.62) and carrying out algebraic calculations (the details are omitted because of lack space) one obtain, the desired energy

$$
\begin{aligned}
E_{n n_{1} n_{2} \mathbf{m}}^{(D)}\left(Z_{1}, Z_{2}, R\right) & =-\frac{Z_{1}^{2}}{2\left(n+\frac{D-3}{2}\right)^{2}}-\frac{Z_{2}}{R}+\frac{3 Z_{2}\left(n+\frac{D-3}{2}\right) \Delta}{2 Z_{1} R^{2}}-\frac{Z_{2}\left(n+\frac{D-3}{2}\right)^{2}}{2 R^{3} Z_{1}^{2}}\left(6 \Delta^{2}-\left(n+\frac{D-3}{2}\right)^{2}+1\right) \\
& +\frac{Z_{2}\left(n+\frac{D-3}{2}\right)^{3}}{16 Z_{1}^{4} R^{4}}\left[Z_{1} \Delta\left(109 \Delta^{2}-39\left(n+\frac{D-3}{2}\right)^{2}-9\left(m_{D-2}+\frac{D-3}{2}\right)^{2}+59\right)(5.73)\right. \\
& \left.-Z_{2}\left(n+\frac{D-3}{2}\right)\left(17\left(n+\frac{D-3}{2}\right)^{2}-3 \Delta^{2}-9\left(m_{D-2}+\frac{D-3}{2}\right)^{2}+19\right)\right]+O\left(R^{-5}\right),
\end{aligned}
$$

where $\Delta=n_{1}-n_{2}$. Some important details for $D=3$ are given in [7] and [19].

Formula (5.73) is the multipole expansion for the electronic term of the electrostatic interaction of the atom $e Z_{1}$ with the remote point charge $Z_{2}$ (e $Z_{1}$-terms). The series of terms which correspond to the atoms $e Z_{2}$ is obtained from (5.73) by replacements

$$
Z_{1} \rightarrow Z_{2}, \quad n \rightarrow n^{\prime}, \quad \Delta \rightarrow \Delta^{\prime}, \quad n_{2} \rightarrow n_{2}^{\prime} .
$$

The adjacent eigenvalues $\lambda_{\mu q}^{\prime(\eta)}$ with respect to $q$ coincide at

$$
\beta=n_{2}^{\prime}-n_{2}
$$

if we do not take into account additional terms $\delta \chi$ and $\delta \chi^{\prime}$. Otherwais said, the adjacent eigenvalues are shifted by an exponentially small value. Therefore the points defined by (5.75) are named the quasi-crossing points. If (5.75) holds, the quasi-crossing of the eigenvalues $\lambda_{\mu q}^{\prime(\eta)}$ and $\lambda_{\mu q+1}^{\prime(\eta)}$ with quantum number $n_{1}=n_{1}^{\prime}$ occurs. This leads to a quasi-crossing of the energy terms

$$
\beta=\left(Z_{2}-Z_{1}\right)(-2 E)^{-1 / 2}=n_{2}^{\prime}-n_{2} .
$$

Thus, the energies of the quasi-crossing form the Coulomb series corresponding to the charge $Z_{2}-Z_{1}$ and the principal quantum number $n_{2}^{\prime}-n_{2}$.

Two series of power expansion for $e Z_{1}$ and $e Z_{2}$-terms correspond to the predominant localizations of the electron of one of the two charges only. The accounts of exponentially small terms with respect to $R$ for $E_{n n_{1} n_{2}}^{(D)} \mathbf{m}(R)$ and $E_{n^{\prime} n_{1}^{\prime} n_{2}^{\prime} \mathbf{m}}^{(D)}(R)$ represent an improvement in accuracy. However, exponential corrections play an important role in the quasi-crossing points. The states of the system are energetic indistinguishable

$$
E_{1}=E_{n n_{1} n_{2}}^{(D)} \mathbf{m}\left(R_{c}\right), \quad E_{2}=E_{n^{\prime} n_{1}^{\prime} n_{2}^{\prime} \mathbf{m}}^{(D)}\left(R_{c}\right),
$$

$$
E_{1}=E_{2}=E\left(R_{c}\right)=-\frac{\left(Z_{2}-Z_{1}\right)^{2}}{2\left(n_{2}^{\prime}-n_{2}\right)^{2}},
$$

in free-form exponential small terms. Here $E_{n n_{1} n_{2}}^{(D)} \mathbf{m}, E_{n^{\prime} n_{1}^{\prime} n_{2}^{\prime} \mathbf{m}}^{(D)}$ are delimited by (5.73) and (5.74), $R_{c}$ is the coordinate of the quasi-crossing following straight from (5.75). The inclusion of exponential terms leads to the splitting of $E_{1}$ and $E_{2}$ into a states in which the electron moves in both wells simultaneously. In this case the minimal distance between energetic terms $\Delta E\left(R_{c}\right)$ (splitting) is explained by the sub-barrier resonance interaction. It is estimated by formula

$$
\Delta E\left(R_{c}\right)=\delta E_{1}\left(R_{c}\right)+\delta E_{2}\left(R_{c}\right)=T \delta\left(R_{c}\right),
$$

where $\delta\left(R_{c}\right)$ is defined by (51). Following [20] and [21] we obtain

$$
T=\frac{\partial E_{1} / \partial n_{2}}{\sqrt{1+\frac{\partial \beta}{\partial E_{1}} \frac{\partial E_{1}}{\partial n_{2}}}}+\frac{\partial E_{2} / \partial n_{2}^{\prime}}{\sqrt{1-\frac{\partial \beta}{\partial E_{2}} \frac{\partial E_{2}}{\partial n_{2}^{\prime}}}} .
$$

All the data which are included in (5.80) can be calculated by (5.73) and (5.74). Then we obtain the splitting of $E_{1}$ and $E_{2}$ 


$$
\begin{aligned}
\Delta E\left(R_{c}\right) & =\left\{\frac{Z_{1}^{2}}{\left(n+\frac{D-3}{2}\right)^{3}}\left[1+\frac{Z_{1}^{2}\left(Z_{2}-Z_{1}\right)}{\left(n+\frac{D-3}{2}\right)^{3}\left(-2 E_{c}\right)^{3 / 2}}\right]^{-1 / 2}\right. \\
& \left.+\frac{Z_{2}^{2}}{\left(n^{\prime}+\frac{D-3}{2}\right)^{3}}\left[1-\frac{Z_{2}^{2}\left(Z_{2}-Z_{1}\right)}{\left(n^{\prime}+\frac{D-3}{2}\right)^{3}\left(-2 E_{c}\right)^{3 / 2}}\right]^{-1 / 2}\right\} \delta_{c} .
\end{aligned}
$$

\section{RULES OF CORRESPONDENCES OF TERMS AS $R \rightarrow 0$ AND $R \rightarrow \infty$}

Under the limit of the united atom $(R \rightarrow 0)$

$$
\xi R / 2 \rightarrow r, \quad \eta \rightarrow \cos \theta .
$$

Then the hyperspheroidal coordinates $\xi, \eta, \beta_{1}, \beta_{2}, \ldots$, $\beta_{D-2}$ are reduced to hyperspherical ( [17] vol. 2) ones $r, \theta, \beta_{1}, \beta_{2}, \ldots, \beta_{D-2}$. Thus

$$
\begin{aligned}
& x_{1}=r \cos \theta, \\
& x_{2}=r \sin \theta \cos \beta_{D-2}, \\
& x_{3}=r \sin \theta \sin \beta_{D-2} \cos \beta_{D-3},
\end{aligned}
$$

$$
\begin{aligned}
& \vdots \\
& x_{D-1}=r \sin \theta \sin \beta_{D-2} \ldots \sin \beta_{2} \cos \beta_{1}, \\
& x_{D}=r \sin \theta \sin \beta_{D-2} \ldots \sin \beta_{2} \sin \beta_{1},
\end{aligned}
$$

where $0 \leq r<\infty, \quad 0 \leq \theta \leq \pi, \quad 0 \leq \beta_{1}<2 \pi, \quad 0 \leq$ $\beta_{k} \leq \pi, \quad k=2,3, \ldots, D-2$.

In this case the radial $\Pi_{m_{D-2} k}^{(D)}(\xi, R)$ and angular part $\Xi_{m_{D-2} q}^{(D)}(\eta, R)$ of the wavefunction of $\left(Z_{1} e Z_{2}\right)_{D}$ system changes to the radial $R_{\mathcal{N} l}^{(D)}(r)$ and angular $Y_{l \mathbf{m}}^{(D)}\left(\theta, \beta_{1}, \beta_{2}, \ldots, \beta_{D-2}\right)$ parts of the solutions of the $D$ dimensional one-centre problem in the hyperspheroidal coordinates with the charge $Z_{1}+Z_{2}$

$$
\Psi_{\mathcal{N} l \mathbf{m}}(r ; 0)=\Pi_{m_{D-2} k}^{(D)}(\xi ; 0) \Xi_{m_{D-2} q}^{(D)}(\eta ; 0) F\left(\beta_{1}, \beta_{2}, \ldots, \beta_{D-2}\right)=R_{\mathcal{N} l}^{(D)}(r) Y_{l \mathbf{m}}^{(D)}\left(\theta, \beta_{1}, \beta_{2}, \ldots, \beta_{D-2}\right),
$$

where

$$
Y_{l \mathbf{m}}^{(D)}\left(\theta, \beta_{1}, \beta_{2}, \ldots, \beta_{D-2}\right)=\mathcal{C}(\sin \theta)^{m_{D-2}} C_{l-m_{D-2}}^{\frac{D-2}{2}+m_{D-2}}(\cos \theta) F\left(\beta_{1}, \beta_{2}, \ldots, \beta_{D-2}\right),
$$

$\mathcal{C}$ is a normalization constant and $[\mathcal{N}, l, \mathbf{m}]$ is a set of hyperspherical quantum numbers.

It is clear from the physical consideration that in the limit of the united atom the terms of the $\left(Z_{1} e Z_{2}\right)_{D}$ problem change to the energy levels of the $D$-dimensional hydrogen atom with the charge $\left(Z_{1}+Z_{2}\right)$. Then

$$
E_{\mathcal{N} l \mathbf{m}}^{(D)}\left(Z_{1}, Z_{2}, 0\right)=-\frac{2\left(Z_{1}+Z_{2}\right)^{2}}{(2 \mathcal{N}+D-3)^{2}}
$$

where $\mathcal{N}=k+l+1$ is the principal quantum number, $l=q+m$.

As $R \rightarrow \infty$ the hyperspheroidal coordinates pass to the $D$-dimensional parabolic coordinates and the functions $\Pi_{m_{D-2} k}^{(D)}(\xi ; R), \quad \Xi_{m_{D-2} k}^{(D)}(\eta ; R)$ change to solutions of the one-centre problem in the $D$-dimensional parabolic coordinates $f_{n_{1} m_{D-2}}(\zeta)$ and $f_{n_{2} m_{D-2}}(\tau)$ [18], respectively. Let the pole of the $D$-dimensional parabolic coordinates system coincide with the left of the hyperspheroidal system. Then we pass by means of limit relations

$$
R(\xi-1) \stackrel{R \rightarrow \infty}{\longrightarrow} \zeta, \quad R(1+\eta) \stackrel{R \rightarrow \infty}{\longrightarrow} \tau,
$$

to the $D$-dimensional parabolic coordinates system [18]

$$
\begin{aligned}
& x_{1}=(\zeta-\tau) / 2, \\
& x_{2}=\sqrt{\zeta \tau} \cos \beta_{D-2}, \\
& x_{3}=\sqrt{\zeta \tau} \sin \beta_{D-2} \cos \beta_{D-3}, \\
& \vdots \\
& x_{D-1}=\sqrt{\zeta \tau} \sin \beta_{D-2} \ldots \sin \beta_{2} \cos \beta_{1}, \\
& x_{D}=\sqrt{\zeta \tau} \sin \beta_{D-2} \ldots \sin \beta_{2} \sin \beta_{1}
\end{aligned}
$$

where $0 \leq \zeta<\infty, 0 \leq \tau<\infty, 0 \leq \beta_{1}<2 \pi, 0 \leq \beta_{k}<\pi$, $k=2,3, \ldots, D-2$.

For $R \rightarrow \infty$ all terms of the system $\left(Z_{1} e Z_{2}\right)_{D}$ fall into two classes: $e Z_{1}$-terms and $e Z_{2}$-terms.

For $e Z_{1}$-terms pass to the electron levels in the field of the isolated charge as $R \rightarrow \infty$ (with the corresponding wave function which are concentrated in neighborhood $\xi \approx 1, \eta=1$ ), and for $e Z_{1}$-terms we have 


$$
\begin{array}{r}
\Psi_{k q} \mathbf{m}(r ; \infty)=\Pi_{m_{D-2} k}^{(D)}(\xi ; \infty) \Xi_{m_{D-2} q}^{(D)}(\eta ; \infty) F\left(\beta_{1}, \beta_{2}, \ldots, \beta_{D-2}\right)= \\
=f_{n_{1} m_{D-2}}(\zeta) f_{n_{2} m_{D-2}}(\tau) F\left(\beta_{1}, \beta_{2}, \ldots, \beta_{D-2}\right)
\end{array}
$$

$$
E_{k q \mathbf{m}}^{(D)}(\infty)=E_{n n_{1} n_{2}}^{(D)} \mathbf{m}=-\frac{2 Z_{1}^{2}}{(2 n+D-3)^{2}},
$$

where $n=n_{1}+n_{2}+m_{D-2}+1$ is the principal quantum number.

For $\left(e Z_{2}\right)$-terms equation (6.89) remains but the limited transition (6.87) changes to

$$
R(\xi-1) \stackrel{R \rightarrow \infty}{\longrightarrow} \zeta, \quad R(1-\eta) \stackrel{R \rightarrow \infty}{\longrightarrow} \tau,
$$

and (6.90) will have the following form

$$
E_{k q \mathbf{m}}^{(D)}(\infty)=E_{n^{\prime} n_{1}^{\prime} n_{2}^{\prime} \mathbf{m}}^{(D)}=-\frac{2 Z_{2}^{2}}{\left(2 n^{\prime}+D-3\right)^{2}},
$$

where $n^{\prime}=n_{1}^{\prime}+n_{2}^{\prime}+m_{D-2}+1$.

For $e Z_{1}$-terms the quantum numbers $n_{1}, n_{2}$ represent the number of zeros of the radial function $f_{n_{1} m_{D-2}}(\zeta)$ and the angular function $f_{n_{2} m_{D-2}}(\tau)$, respectively. Analogous $n_{1}^{\prime}, n_{2}^{\prime}$ have the same meaning for $\left(e Z_{2}\right)$-terms. The sets of the $D$-dimensional parabolic quantum numbers $\left[n, n_{1}, n_{2}, \mathbf{m}\right],\left[n^{\prime}, n_{1}^{\prime}, n_{2}^{\prime}, \mathbf{m}\right]$, differ from the sets of hyperspherical quantum numbers $[\mathcal{N}, l, \mathbf{m}]$ and the initial of the hyperspheroidal quantum numbers $[k, q, \mathbf{m}]$. However, they are linked with them by unambiguous rules of the correspondence, sometimes named the correlation diagrams of terms.

For the radial numbers to be valid

$$
k=n_{1}=n_{1}^{\prime},
$$

that is a consequence of a theorem for the ShturmLiouville problem on the conservation of the number of zeros of a solution which depends continuously on the parameter.

The relation for angular quantum numbers $q, n_{2}$ and $n_{2}^{\prime}$ is more complicated, in particular for $Z_{2}>Z_{1}$. We present it without the proof (just as for [7]).

For $e Z_{1}$-terms

$$
q=\left\{\begin{array}{lll}
2 n_{2}+\left(n+\frac{D-3}{2}\right) \frac{Z_{2}-Z_{1}}{Z_{1}}, & \text { if } & \left(n+\frac{D-3}{2}\right) \frac{Z_{2}-Z_{1}}{Z_{1}}=\text { integer } \\
2 n_{2}+1+\operatorname{Ent}\left(\left(n+\frac{D-3}{2}\right) \frac{Z_{2}-Z_{1}}{Z_{1}}\right), & \text { if } & \left(n+\frac{D-3}{2}\right) \frac{Z_{2}-Z_{1}}{Z_{1}} \neq \text { integer }
\end{array}\right.
$$

and for the $e Z_{2}$-terms

$$
q=\left\{\begin{array}{llc}
n_{2}^{\prime}, & \text { if } \quad n_{2}^{\prime}<\left(n^{\prime}+\frac{D-3}{2}\right) \frac{Z_{2}-Z_{1}}{Z_{1}} \\
n_{2}^{\prime}+1+\operatorname{Ent}\left(n_{2}^{\prime}-\left(n^{\prime}+\frac{D-3}{2}\right) \frac{Z_{2}-Z_{1}}{Z_{1}}\right), & \text { if } \quad n_{2}^{\prime} \geq\left(n^{\prime}+\frac{D-3}{2}\right) \frac{Z_{2}-Z_{1}}{Z_{1}} .
\end{array}\right.
$$

Here Ent $(x)$ is the greatest integer $\leq x$.

There is a connection of the hyperspherical quantum number $[\mathcal{N}, l, \mathbf{m}]$ with the $D$-dimensional parabolic quantum numbers $\left[n, n_{1}, n_{2}, \mathbf{m}\right],\left[n^{\prime}, n_{1}^{\prime}, n_{2}^{\prime}, \mathbf{m}\right]$ if the electron is found at the nuclei $Z_{1}\left(Z_{2}>Z_{1}\right)$

$$
l=\left\{\begin{array}{c}
2 n_{2}+\left|m_{D-2}\right|+\left(n+\frac{D-3}{2}\right) \frac{Z_{2}-Z_{1}}{Z_{1}}, \\
\text { if }\left(n+\frac{D-3}{2}\right) \frac{Z_{2}-Z_{1}}{Z_{1}}=\text { integer, } \\
n_{2}+\left|m_{D-2}\right|+1+\operatorname{Ent}\left(n_{2}+\left(n+\frac{D-3}{2}\right) \frac{Z_{2}-Z_{1}}{Z_{1}}\right), \\
\text { if } \quad\left(n+\frac{D-3}{2}\right) \frac{Z_{2}-Z_{1}}{Z_{1}} \neq \text { integer, }
\end{array}\right.
$$

$$
\mathcal{N}=\left\{\begin{aligned}
n+n_{2}+ & \left(n+\frac{D-3}{2}\right) \frac{Z_{2}-Z_{1}}{Z_{1}} \\
& \text { if } \quad\left(n+\frac{D-3}{2}\right) \frac{Z_{2}-Z_{1}}{Z_{1}}=\text { integer } \\
n_{2}+1+ & \operatorname{Ent}\left(n_{2}+\left(n+\frac{D-3}{2}\right) \frac{Z_{2}-Z_{1}}{Z_{1}}\right) \\
& \text { if } \quad\left(n+\frac{D-3}{2}\right) \frac{Z_{2}-Z_{1}}{Z_{1}} \neq \text { integer }
\end{aligned}\right.
$$

and similarly for the electron which is found at the nuclei $Z_{2}\left(Z_{2}>Z_{1}\right)$

$$
\begin{gathered}
l=\left\{\begin{aligned}
n_{2}^{\prime}+\left|m_{D-2}\right|, \\
\text { if } n_{2}<\left(n^{\prime}+\frac{D-3}{2}\right) \frac{Z_{2}-Z_{1}}{Z_{1}}, \\
n_{2}^{\prime}+\left|m_{D-2}\right|+1+\operatorname{Ent}\left(n_{2}^{\prime}+\left(n^{\prime}+\frac{D-3}{2}\right) \frac{Z_{2}-Z_{1}}{Z_{1}}\right), \\
\text { if } n_{2}^{\prime} \geq\left(n^{\prime}+\frac{D-3}{2}\right) \frac{Z_{2}-Z_{1}}{Z_{1}},
\end{aligned}\right. \\
\mathcal{N}= \begin{cases}n^{\prime}, & \text { if } \quad n_{2}<\left(n^{\prime}+\frac{D-3}{2}\right) \frac{Z_{2}-Z_{1}}{Z_{1}} \\
n^{\prime}+1+\operatorname{Ent}\left(n_{2}^{\prime}+\left(n^{\prime}+\frac{D-3}{2}\right) \frac{Z_{2}-Z_{1}}{Z_{1}}\right), & \text { if } \quad n_{2}^{\prime} \geq\left(n^{\prime}+\frac{D-3}{2}\right) \frac{Z_{2}-Z_{1}}{Z_{1}} .\end{cases}
\end{gathered}
$$


For $Z_{1}=Z_{2}$ the separation of the eigenvalues $E_{n n_{1} n_{2}} \mathbf{m}(R)$ does not exist for $e Z_{1}$-terms and $e Z_{2}$-terms, as $R \rightarrow \infty$. The angular function $\Xi_{m_{D-2} q}^{(D)}(\eta ; \infty)$ is of the same order at both centres, and near each of them is concentrated on the equal number of zeros, i. e., $n_{2}=n_{2}^{\prime}$.

\section{CONCLUSION}

We obtained asymptotic expansion for eigenfunctions and eigenvalues of the energy of the Schrödinger equations for the $\left(Z_{1} e Z_{2}\right)_{D}$ problem at large intercenter distances.

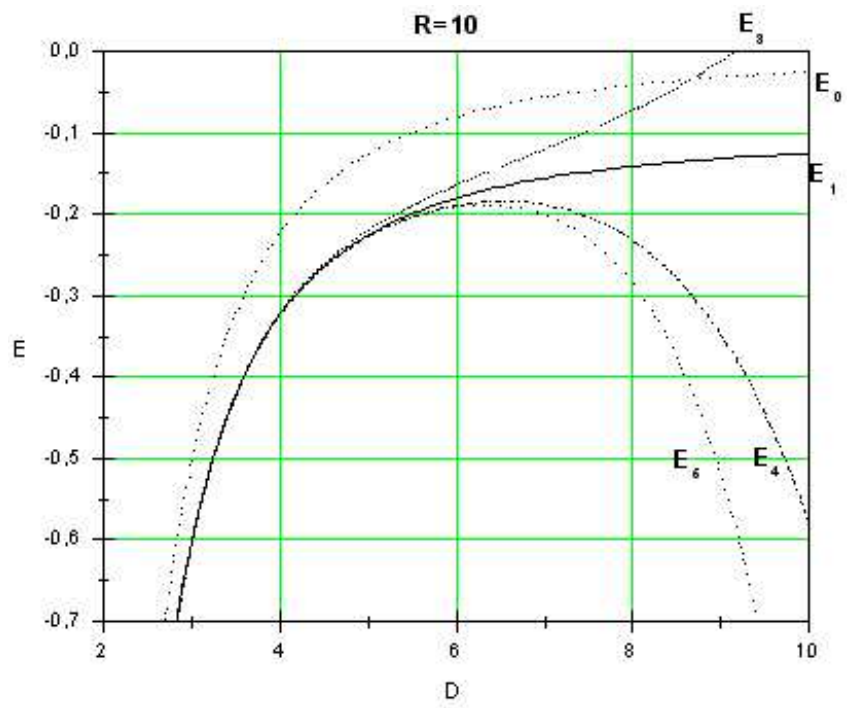

Fig. 1. Dependence $E_{k}$ for the ground state of $H_{2}^{+}$at the value of the intercentral distance $R=10$ a.u. on the dimension of space $D$.

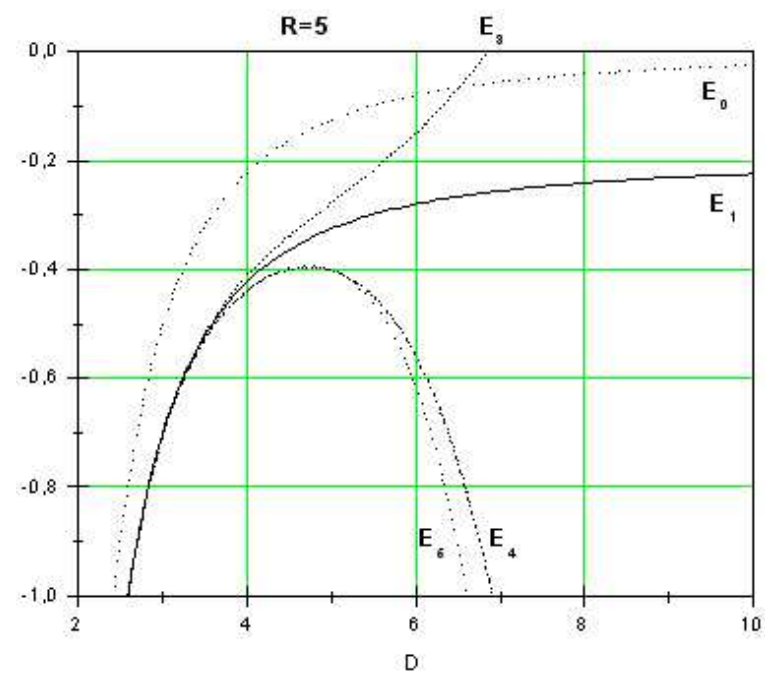

Fig. 2. Dependence $E_{k}$ for the ground state of $H_{2}^{+}$at the value of the intercentral distance $R=5$ a.u. on the dimension of space $D$.

Figures 1 and 2 show the dependence of different $E_{k}$ for the ground state of a molecular ion of hydrogen $\mathrm{H}_{2}^{+}$ $\left(Z_{1}=Z_{2}=1, n_{1}=n_{2}=m_{D-2}=0\right)$ on the dimension of the space $D$, at the values of the centre separation $R=10$ and $R=5$ a.u., respectively. Here $E_{k}$ is the energy for the ground state of $\mathrm{H}_{2}^{+}$with the accuracy to $O\left(R^{-k}\right)$ which follows from (5.73). It is clearly visible from the figures that in the energy series (5.73) divergence begins from the value of $D=O(1)$ that is conditioned by relation (3.28) for $\mu$.

\section{ACKNOWLEDGEMENTS}

This paper is supported by the project MVTS of the Ministry of Education of Slovak Republic.
[1] P. Ehrenfest, Proc. Amsterdam Acad. 20, 200 (1917).

[2] G. E. Gorelik, Razmernost' prostranstva (Space dimension) (MGU, Moskow, 1983) (in Russian).

[3] C. A. Tsipis, New Methods in Quantum Theory (Kluwer Academic Publ., Dordrecht, 1996).

[4] C. M. Bender, L. D. Mlodinov, N. Papanicolaou, Phys. Rev. A 25, 1305 (1982).

[5] V. S. Popov, A. V. Sergeev, Zh. Eksp. Teor. Fiz. 105, 568 (1994).

[6] D. R. Herschbach, Dimensional Scaling in Chemical Phys (Kluwer Academic Publ., Dordrecht, 1993).

[7] I. V. Komarov, L. I. Ponomarev, S. Yu Slavyanov, Spheroidal and Coulomb spheroidal functions (Nauka, Moskva, 1976) (in Russian).

[8] S. Yu. Slavyanov, W. Lay, Special function: A United Theory Based on Singularities (Oxford University Press, New York, 2000).

[9] A. Ishkhanyan, J. Phys. A: Math. Gen. 38, L491 (2005).

[10] V. V. Rubish, V. Yu. Lazur, V. M. Dobosh, S. Chalupka, M. Salak, J. Phys. A: Math. Gen. 37, 9951 (2004).
[11] L. G. Mardoyan, A. N. Sissakian, V. M. Ter-Antonyan, Mod. Phys. Lett. A 14, 1303 (1999).

[12] L. G. Mardoyan, A. N. Sissakian, V. M. Ter-Antonyan, Teor. Mat. Fiz. 123, 44 (2000).

[13] C. N. Yang, J. Math. Phys. 19, 320 (1978).

[14] S. A. Teukolsky, Phys. Rev. Lett. 29, 1114 (1972).

[15] N. F. Truskova, J. Yad. Fiz. 36, 790 (1982).

[16] H. Buckholz, The Confluent Hypegeomitric Functions (Springer Verlag, Berlin, 1969).

[17] H. Bateman, A. Erdelyi, Higher transcendental functions Vols. 1-3 (Mc Graw-Hill Book Company, Inc. New York, 1958).

[18] S. P. Alliluev, V. S. Popov, Zh. Eksp. Teor. Fiz. 104, 3569 (1993).

[19] J. D. Power, Phil. Trans. R. Soc. London A 274, 663 (1973).

[20] L. I. Ponomarev, Zh. Eksp. Teor. Fiz. 55, 1836 (1968).

[21] I. V. Komarov, E. A. Solovyov, Teor. Mat. Fiz. 40, 130 (1979). 


\section{ЗАДАЧА ДВОХ КУЛОНІВСЬКИХ ЦЕНТРІВ У КВАНТОВІЙ МЕХАНІЩІ.} ВПЛИВ РОЗМІРНОСТИ

Д. І. Бондар ${ }^{1}$, В. Ю. Лазур ${ }^{1}$, І. М. Шваб ${ }^{1}$, С. Халупка ${ }^{2}$

${ }^{1}$ Фізичний факультет Ужгородсъкого начіонального університету, вул. Волошина, 54, Ужсгород, 88000, Україна

${ }^{2}$ Інститут фізики, Університет ім. П. Й. Шафарика, вул. Ясенна, 5, 04154 Кошіче, Словацька Республіка

Розглянуто задачу двох кулонівських центрів. Проаналізовано вплив розмірности простору $(D \geq 3)$. Побудовано асимптотичні розв'язки рівняння Шредингера з двома кулонівськими потенціялами в гіперсфероїдальній системі координат. Побудовані розв'язки виражаються через конфлюентні функції Гойна, частковим випадком яких є кулоновські сфероїдальні функції. Розглянуто асимптотичний розклад для енергії цієї системи при великих міжцентрових відстанях і вивчено їхні основні властивості. 